\section{Check for updates}

Cite this: Soft Matter, 2018, 14,901

Received 25th August 2017, Accepted 26th October 2017

DOI: $10.1039 / c 7 s m 01718 a$

rsc.li/soft-matter-journal

\title{
Spherical network contraction forms microtubule asters in confinement $\dagger$
}

\author{
Michael P. N. Juniper, ${ }^{a}$ Marian Weiss, ${ }^{\text {bc }}$ Ilia Platzman, ${ }^{\text {bc }}$ Joachim P. Spatz $^{\text {bc }}$ and \\ Thomas Surrey $(\mathbb{D}$ *a
}

\begin{abstract}
Microtubules and motor proteins form active filament networks that are critical for a variety of functions in living cells. Network topology and dynamics are the result of a self-organisation process that takes place within the boundaries of the cell. Previous biochemical in vitro studies with biomimetic systems consisting of purified motors and microtubules have demonstrated that confinement has an important effect on the outcome of the self-organisation process. However, the pathway of motor/microtubule self-organisation under confinement and its effects on network morphology are still poorly understood. Here, we have investigated how minus-end directed microtubule cross-linking kinesins organise microtubules inside polymer-stabilised microfluidic droplets of well-controlled size. We find that confinement can impose a novel pathway of microtubule aster formation proceeding via the constriction of an initially spherical motor/microtubule network. This mechanism illustrates the close relationship between confinement, network contraction, and aster formation. The spherical constriction pathway robustly produces single, well-centred asters with remarkable reproducibility across thousands of droplets. These results show that the additional constraint of well-defined confinement can improve the robustness of active network self-organisation, providing insight into the design principles of selforganising active networks in micro-scale confinement.
\end{abstract}

${ }^{a}$ The Francis Crick Institute, 1 Midland Road, London, NW1 1AT, UK.

E-mail: thomas.surrey@crick.ac.uk

${ }^{b}$ Department of Cellular Biophysics, Max Planck Institute for Medical Research, Jahnstraße 29, 69120, Germany

${ }^{c}$ Department of Biophysical Chemistry, University of Heidelberg,

Im Neuenheimer Feld 253, 69120 Heidelberg, Germany

$\dagger$ Electronic supplementary information (ESI) available: Movie 1: Microtubule vortex formation in surfactant stabilised droplets. Conditions as in Fig. 2a: 0.9\% surfactant in oil, $200 \mathrm{nM}$ mCherry-kinesin-14 and $40 \mu \mathrm{M}$ Alexa647-tubulin in the droplet. Temperature was $34{ }^{\circ} \mathrm{C}$. Microtubules are shown in red, motor protein in green. Movie 2: Distorted microtubule aster formation in surfactant stabilised droplets. Conditions as in Fig. 2 b: $0.9 \%$ surfactant in oil, $400 \mathrm{nM}$ mCherrykinesin-14 and $40 \mu \mathrm{M}$ Alexa647-tubulin in the droplet. Temperature was $34{ }^{\circ} \mathrm{C}$. Microtubules are shown in red, motor protein in green. Movie 3: Centred microtubule aster formation in surfactant stabilised droplets. Conditions as in Fig. 2c: $1.8 \%$ surfactant in oil, $200 \mathrm{nM}$ mCherry-kinesin-14 and $40 \mu \mathrm{M}$ Alexa647tubulin in the droplet. Temperature was $34{ }^{\circ} \mathrm{C}$. Microtubules are shown in red, motor protein in green. Movie 4: Movie illustrating the process of averaged kymograph generation. The movie shows 360 individual kymographs of aster formation along droplet diameters separated by $1^{\circ}$. Conditions as in Fig. $2 \mathrm{c}$ and Movie 3. Movie 5: Repeatability of aster formation by the network constriction mechanism. Three time sequences are shown: first, aster formation at $30{ }^{\circ} \mathrm{C}$; second, microtubule depolymerisation and aster disappearance after cooling to $4{ }^{\circ} \mathrm{C}$; third, aster reformation after reheating to $30{ }^{\circ} \mathrm{C}$. Surfactant and protein concentrations as in Fig. $2 \mathrm{c}$ and Movie 3. Microtubules are shown in red, motor protein in green. See DOI: 10.1039/c7sm01718a

\section{Introduction}

The internal structure of living cells depends on the organisation of the cytoskeleton within the cell boundary. Microtubules are dynamic tubular polymers of the cytoskeleton that interact with a multitude of associated proteins. Among these are several types of molecular motor that can form active cross-links between microtubules to create a dynamic network, which critically contributes to the overall organisation of the cytoskeleton. ${ }^{1-5}$ Depending on the cellular state, the microtubule cytoskeleton can re-organise into remarkably different architectures with different biological functions. ${ }^{6,7}$ In interphase, the microtubule cytoskeleton is important for providing a polar scaffold for intracellular cargo transport, and during cell division it is essential for mitotic spindle assembly and chromosome segregation. ${ }^{1,2,6,7}$ The molecular mechanisms determining the dynamic organisation of the complex microtubule network within the boundary of a eukaryotic cell are however only poorly understood.

Biomimetic systems of purified cytoskeletal components provide reduced complexity relative to their living counterparts. This offers the opportunity to gain insight into the mechanisms underlying the basic behaviour of active cytoskeletal networks, ${ }^{3,8}$ as well as addressing more fundamental questions about the design principles of active matter systems. In previous work, 
in vitro experiments with microtubules and motors led to the formation of large contracting networks or multiple microtubule asters or vortices, ${ }^{9-12}$ or, in the additional presence of depletion forces, to the generation of active gels or nematic liquid crystals consisting of extensile microtubule bundles. ${ }^{13-15}$ Related observations were also made for microtubules organised by motors in more complex cell extracts. ${ }^{16,17}$ The role of physical confinement on self-organising motor/microtubule systems is less studied, despite the importance of this constraint for the organisation of the cytoskeleton within cells, but some studies exist.

Pioneering work visualised individual dynamic microtubules growing from purified tubulin in small unilamellar vesicles. ${ }^{18,19}$ Later, microtubule asters nucleated from purified centrosomes (microtubule nucleating organelles) were studied in micro-fabricated chambers, investigating the conditions for the centering of aster in these chambers. ${ }^{20-22}$ Recently centrosomenucleated asters were also encapsulated in lipid-monolayered droplets in oil. ${ }^{23}$ In the absence of centrosomes, and in the presence of motor proteins and a depletion force by a nonadsorbing polymer, active nematics were observed at the inner surface of large unilamellar liposomes formed by short stabilised microtubules that were cross-linked and slid relative to each other by molecular motors. ${ }^{24}$ Self-organisation of microtubules and motors was also studied in the absence of depletion forces in micro-fabricated chambers ${ }^{10}$ and in lipid monolayer droplets surrounded by oil that ranged in size from typically 5 to $50 \mu \mathrm{m}$ in diameter. ${ }^{25}$ In these experiments, microtubules nucleated and grew within their confined space, followed by motor protein cross-linking, sliding and organisation. In micro-chambers, initially forming microtubule asters transformed into vortices as more and/or longer microtubules grew. ${ }^{10}$ In the much smaller droplets most microtubules organised into bent bundles at the droplet boundary, whereas in droplets larger than about $40 \mu \mathrm{m}$ the motors were able to cluster microtubule ends into a single focal point, leading to the formation of a microtubule aster. ${ }^{25}$ The dependence of aster formation on droplet size was explained by considering that an aster with a distinct typical microtubule length will only fit into droplets where the induced microtubule bending is not too strong. ${ }^{10,25}$ This is in agreement with a study performed using cell extract. ${ }^{26}$ In previous work with purified motors and microtubules in droplets surrounded by oil, the droplet size was not controlled and only some droplets were large enough to allow aster formation having prevented a detailed study of the pathway of aster self-organisation under confinement. ${ }^{25}$

Here, we extend this previous work by producing relatively large droplets of uniform size using microfluidic droplet formation. Using polymer surfactants instead of phospholipids allowed for improved tubulin activity compared to previous experiments. ${ }^{25,27}$ We find that the mechanism of aster formation is determined by both the biochemistry of the encapsulated solution and the chemistry of the droplet boundary. Under optimal conditions, single asters form robustly in thousands of droplets. We observe a novel pathway of aster formation based on the contraction of a spherical network which arises due to the imposed confinement.

\section{Materials and methods}

\section{Protein biochemistry}

Pig brain tubulin was purified and labelled with Alexa647- $N$ hydroxy succinimidylester (Sigma) using standard procedures, ${ }^{28,29}$ flash frozen in aliquots and stored in liquid nitrogen. mCherrylabelled Xenopus laevis kinesin-14 $\mathrm{XCTK}^{30}$ was prepared as described previously, ${ }^{25}$ flash frozen in aliquots and stored in liquid nitrogen.

\section{Droplet-based microfluidics}

Microfluidic devices for droplet production using a flow focusing geometry ${ }^{31}$ were designed using QCADpro (RibbonSoft, Switzerland). The corresponding photomasks were printed on chrome-coated soda lime glass (JD-Photodata, UK). The photolithography process was performed as described before: ${ }^{32,33}$ a negative photoresist (SU8-3025, MicroChem, USA) was spin-coated onto silicon wafers at $2500 \mathrm{rpm}$, resulting in uniform coating of $30 \mu \mathrm{m}$ thickness. Wafers were then transferred to a hot plate for soft bake at $65{ }^{\circ} \mathrm{C}$ for five minutes, then ramped slowly to $95{ }^{\circ} \mathrm{C}$ and held for 15 minutes. Following this, the photoresist and the corresponding photomask were exposed for 7.5 seconds to UV light in a mask aligner (MJB4, SÜSS MicroTec, Germany). For the post exposure bake, the wafers were placed for 1 minute on a hot plate at $65{ }^{\circ} \mathrm{C}$ and 5 minutes at $95{ }^{\circ} \mathrm{C}$. The remaining resist was removed with mr-DEV 600 developer (MicroChemicals, Germany). The final hard bake was carried out in an oven at $150{ }^{\circ} \mathrm{C}$ for 15 minutes.

Soft lithography was performed as described before: ${ }^{34,35}$ briefly, PDMS (polydimethylsiloxane, Sylgard 184, Dow Corning, USA) was prepared by mixing the polymerization catalyst with the cross-linking oligomer in a $1: 9(\mathrm{w} / \mathrm{w})$ ratio. The mixed elastomer was degassed using vacuum, poured over the silicon wafer and cured at $65{ }^{\circ} \mathrm{C}$ for 2 hours. After curing, the flexible PDMS mold was cut off and peeled off the wafer. The injection and outlet holes with a diameter of $0.75 \mathrm{~mm}$ were punched using a biopsy puncher (World Precision Instruments, USA). The device was cleaned with ethanol and activated together with a $24 \times 60 \mathrm{~mm}$ coverslip (\#1, Carl Roth, Germany) in an oxygen plasma (PVA TePla 100, PVA TePla, Germany) at 0.45 mbar, $200 \mathrm{~W}$ for $20 \mathrm{~s}$. After activation, the PDMS device was pressed on the coverslip and heated for 2 hours at $65{ }^{\circ} \mathrm{C}$. In order to make the device hydrophobic, Sigmacote (Sigma-Aldrich, Germany) was flushed through the micro channels of the device.

\section{Internal droplet solution}

The droplet solution was prepared by mixing on ice: $20 \mu \mathrm{l}$ SOB2x buffer (40 mM K-PIPES pH 6.8, $100 \mathrm{mM} \mathrm{KCl,} 67 \mathrm{mM}$ glucose, $10 \mathrm{mM}$ ATP, $3 \mathrm{mM}$ GTP, $4.5 \mathrm{mM} \mathrm{MgCl}_{2}$, 2 mM EGTA, $2 \mathrm{mM}$ 2-mercaptoethanol, $0.5 \mathrm{mg} \mathrm{ml}^{-1} \beta$-casein), $1 \mu \mathrm{l}$ antioxidant mix (20 mg ml${ }^{-1}$ glucose oxidase, $10 \mathrm{mg} \mathrm{ml}^{-1}$ catalase in BRB80 (80 mM PIPES pH 6.8, $1 \mathrm{mM} \mathrm{MgCl}_{2}, 1 \mathrm{mM}$ EGTA), pre-centrifuged at $21000 \mathrm{~g}$ for $15 \mathrm{~min}$ ), $9.76 \mu \mathrm{l}$ tubulin $\mathrm{mix}$ (10.75 $\mathrm{mg} \mathrm{ml}^{-1}$ unlabelled tubulin, $5.57 \mathrm{mg} \mathrm{ml}^{-1}$ Alexa647labelled tubulin (25\% labelling ratio) in BRB80), $4 \mu \mathrm{l}$ of motor mix (1 volume $1 \mathrm{mg} \mathrm{ml} \mathrm{m}^{-1}$ mCherry-kinesin-14 mixed with 4 volumes motor buffer (25 mM HEPES pH 7.2, $250 \mathrm{mM} \mathrm{KCl}$, 
$2 \mathrm{mM} \mathrm{MgCl}$, $1 \mathrm{mM}$ 2-mercaptoethanol)), and $5.24 \mu \mathrm{l}$ water (total volume $40 \mu \mathrm{l}$ ). The final mix was centrifuged at $17000 \mathrm{~g}$ for $5 \mathrm{~min}$ and kept on ice until used (as internal phase) to make droplets. Final protein concentrations were $40 \mu \mathrm{M}$ tubulin dimer (final Alexa647 labelling ratio $\sim 9 \%$ ) and $200 \mathrm{nM}$ mCherry-kinesin-14 (monomer concentration). For experiments with $400 \mathrm{nM}$ motor protein, motor mix consisted of 2 volumes mCherry-kinesin-14 and 3 volumes motor buffer.

\section{Droplet production}

For the surfactant/oil mixture, first, a $10 \% \mathrm{w} / \mathrm{w}$ stock solution of RAN-008 fluorosurfactant (according to the manufacturer, a non-ionic PFPE-PEG-PEPE triblock copolymer surfactant, RAN Biotechnologies, USA) in HFE7500 (3M, USA) perfluorinated oil was prepared. Then an $18 \%$ or $9 \% \mathrm{v} / \mathrm{v}$ dilution of this stock solution in HFE7500 was made to generate a $1.8 \%$ or $0.9 \%$ mixture of surfactant in oil, respectively. This mixture was briefly vortexed before being used as the external phase in droplet making. Droplets of about $145 \mu \mathrm{m}$ diameter in the $x / y$ plane were produced in a cold room at $4{ }^{\circ} \mathrm{C}$ using the microfluidic droplet maker and Aladdin AL-1000 (World Precision Instruments, USA) precision syringe pumps with flow rates of $320 \mu \mathrm{h} \mathrm{h}^{-1}$ for the external oil phase and $280 \mu \mathrm{lh}^{-1}$ for the internal (aqueous) proteincontaining solution. Droplets were collected in $10 \mu \mathrm{l}$ pipette tips in an Eppendorf tube and kept on ice.

\section{Fluorescence microscopy}

To prepare flow chambers for microscopy, $25 \times 75 \mathrm{~mm}$ glass slides and $22 \mathrm{~mm} \times 22 \mathrm{~mm}$ glass cover slips (no. 1.5) were sonicated in $20 \%$ ethanol, rinsed with MilliQ water, sonicated in MilliQ water, dried and coated with Aquapel, which is a fluorophilic and hydrophobic chemical which bonds covalently to glass. A flow chamber of $\sim 15 \mu \mathrm{l}$ volume was constructed from a slide and coverslip using TESA double sided tape, placed on a metal block on ice and filled with ice cold HFE7500. Droplets were then drawn in using a tissue, followed by sealing the chamber with clear nail varnish. Microtubule polymerisation was started by transferring the flow chamber to a temperature controlled box heated to $30-34{ }^{\circ} \mathrm{C}$ around a $3 \mathrm{i}$ Marianas spinning disk confocal fluorescence microscope. It is based on a Zeiss Axio Observer $\mathrm{Z} 1$ and is equipped with a $20 \times / \mathrm{NA} 0.8$ plan apo objective, a Yokogawa CSU-X1 spinning disk system and a Hamamatsu Orcaflash 4.0 CMOS camera controlled by 3i SlideBook 6 software. Movies of the central droplet plane parallel to the glass surface were recorded for $45 \mathrm{~min}$ at two frames per min with $100 \mathrm{~ms}$ and $200 \mathrm{~ms}$ exposure times for the $640 \mathrm{~nm}$ and $561 \mathrm{~nm}$ laser respectively.

For experiments where microtubules were depolymerised and repolymerised by temperature shifts (Fig. 5), depolymerisation was induced by placing onto the flow chamber a Northbridge cooler connected to a liquid circulating pump (Lauda Eco RE 415) containing $\sim 5 \%$ ethanol in water at $2{ }^{\circ} \mathrm{C}$. This shifted the temperature in the flow chamber to $4{ }^{\circ} \mathrm{C}$ within $\sim 2 \mathrm{~min}$. Removing the cooler allowed the temperature in the chamber to increase back to $30{ }^{\circ} \mathrm{C}$ within $\sim 6 \mathrm{~min}$. For these experiments, movies were recorded during the cooling phase for $15 \mathrm{~min}$ at 12 frames per minute.

Three-dimensional images were created by making images at multiple confocal planes separated by $1 \mu \mathrm{m}$, which were then reconstructed in ImageJ to produce slices through the $x z$ and $y z$ planes.

\section{Kymograph analysis}

Merged colour time series and kymographs were produced using Fiji. To produce averaged diameter kymographs, a line across the diameter of a droplet was drawn, and the 'Radial Reslice' tool was used to generate a stack of 360 kymographs for each diameter line rotated by 1 degree increments around the droplet centre. ' $\mathrm{Z}$ project' averaged these kymographs into one image. Network constriction speed was manually extracted from the initially approximately linear phase of constriction in the five kymographs shown in Fig. 4c.

\section{Droplet size}

The droplet size distribution was analyzed using Fiji's Analyze Particle plugin. Recorded fluorescence images of the droplets produced in the presence of $1.8 \%$ surfactant were converted
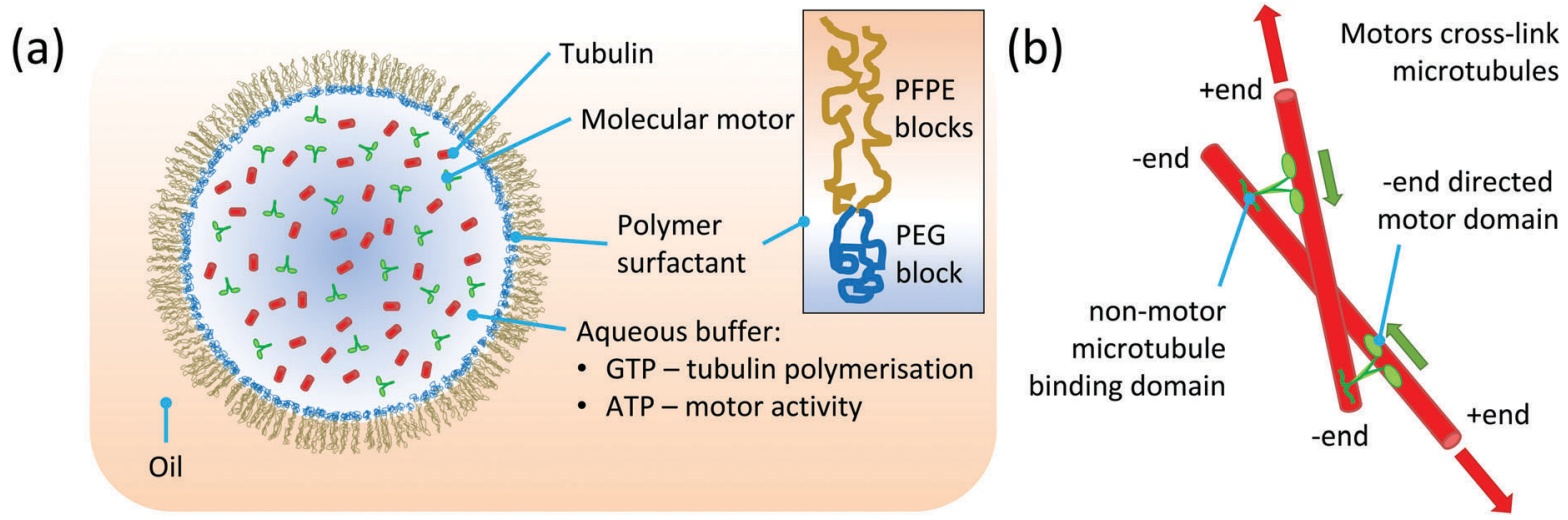

Fig. 1 Schematic of confined active self-organising motor/microtubule system. (a) Schematic of a tri-block copolymer-stabilised protein containing droplet. Encapsulated are tubulin, the motor kinesin-14, GTP (for microtubule polymerisation) and ATP (for motor activity). (b) Schematic view of kinesin14 cross-linking motor activity. Motor walks towards the minus ends of microtubules as it hydrolyses ATP, and transports an other microtubule by binding to it in a non-ATP dependent manner. 
into binary images with optimized thresholds. The particle analyzer was applied to these images and identified the area of the single droplets, from which the diameter in the $x / y$ plane was calculated. A total amount of 343 droplets was detected, resulting in a mean diameter of $145.3 \mu \mathrm{m}$ with a standard deviation of $5.7 \mu \mathrm{m}$. The corresponding coefficient of variation is $4 \%$, in good agreement with previous studies. ${ }^{36,37}$

\section{Results and discussion}

To study how confinement can influence the mechanism of self-organisation of motors and microtubules, we further developed a recent realisation of an in vitro self-organisation assay in which purified motors and tubulin were manually encapsulated in phospholipid-monolayered droplets surrounded by mineral oil. ${ }^{25}$ In the new experimental set-up, we used dropletbased microfluidics to reproducibly generate monodisperse droplets with about $145 \mu \mathrm{m}$ diameter (Methods), a size expected to be considerably larger than typical microtubule lengths under our in vitro conditions and therefore optimal for our study here. ${ }^{25}$ We furthermore changed the chemistry of the dropletsurrounding solution. We replaced mineral oil by perfluorinated oil, and phospholipids by a non-ionic PFPE-PEG-based copolymer surfactant (Methods) that provides a polyethylene glycol (PEG) layer on the inner surface of the droplet (Fig. 1a). (a)

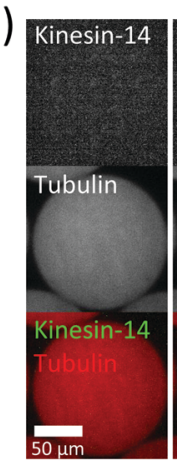

$\mathrm{t}=0$ mins

(b)

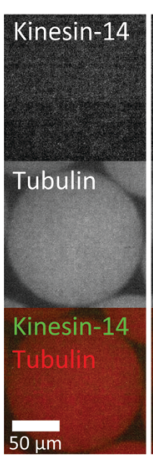

$\mathrm{t}=0$ mins

(c)

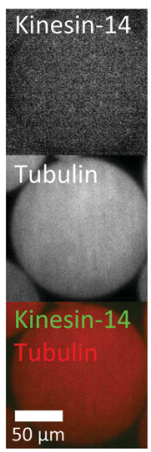

$\mathrm{t}=0$ mins

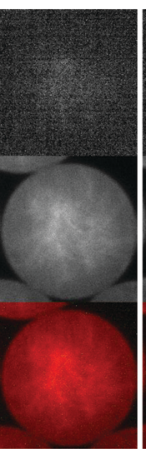

6.5

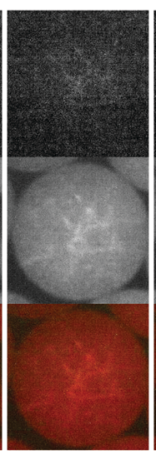

4.5

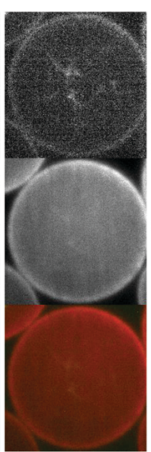

3.5

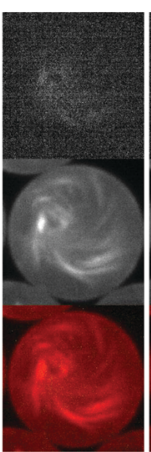

10.5

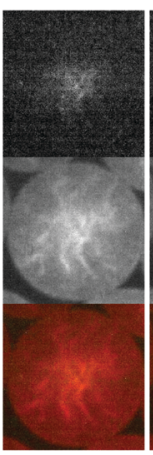

10.5

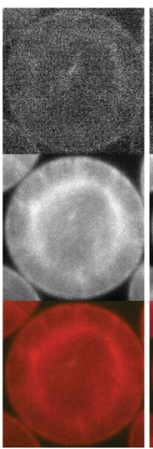

10.5

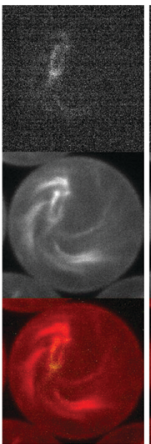

16.5

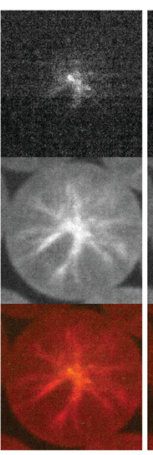

19

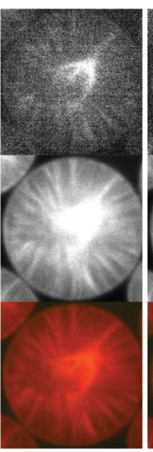

15.5

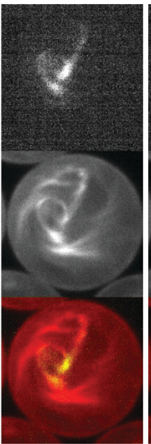

26

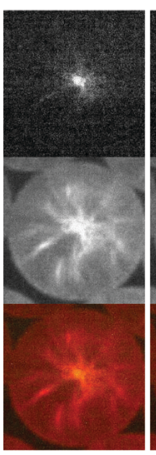

22.5

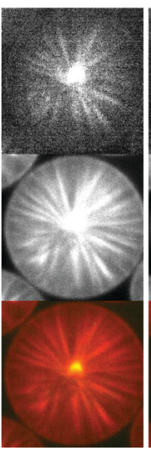

20

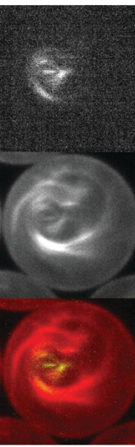

34.5

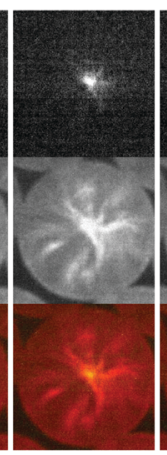

30

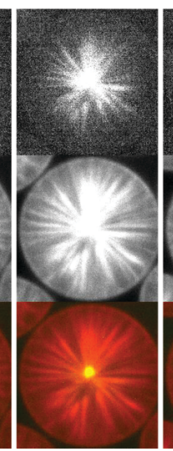

23.5

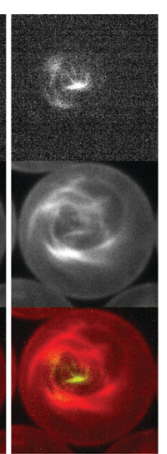

45

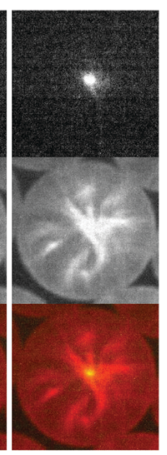

45

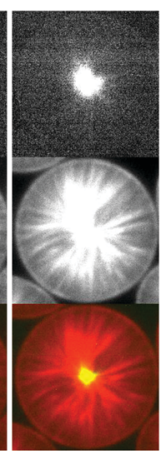

45

Fig. 2 Microtubule structures in surfactant-stabilised droplets produced using a microfluidic droplet generator. (a-c) Confocal fluorescence microscopy time series of self-organising motors (green in merge) and dynamic microtubules (red in merge) at different surfactant and mCherry-kinesin-14 concentrations: (a) $0.9 \%$ surfactant in oil, 200 nM motor; (b) $0.9 \%$ surfactant in oil, 400 nM motor; (c) $1.8 \%$ surfactant in oil, 200 nM motor. The Alexa647tubulin concentration was always $40 \mu \mathrm{M}$. Microtubule polymerisation was started by shifting the temperature to $34{ }^{\circ} \mathrm{C}$. See also Movies $1-3$ (ESI $\dagger$ ). 
Such PEG layers are often used to minimise interactions of proteins with surfaces in microscopy or microfluidics experiments. ${ }^{38-41}$ The modified chemistry at the droplet boundary allowed us to omit microtubule stabilising agents (taxol, glycerol) from the encapsulated solution that were previously required to stimulate efficient microtubule nucleation in droplets ${ }^{25,42}$ (Methods). Omitting these stabilising agents is expected to allow microtubules to remain dynamic throughout the experiment.

Using our modified method of droplet generation, we encapsulated purified mCherry-tagged Xenopus laevis kinesin14 XCTK2 $^{30}$ ) and Alexa647-labelled tubulin, together with GTP and ATP (Methods). Nucleation of microtubules was started by a temperature shift from 0 to $34{ }^{\circ} \mathrm{C}(t=0 \mathrm{~min})$ allowing then the minus end-directed kinesin- $14^{9,43,44}$ to cross-link and slide the growing microtubules relative to each other ${ }^{9,45}$ (Fig. 1b). This can lead to large-scale organisation of a dynamically interconnected network, as observed previously for this motor in the absence and presence of confinement. ${ }^{9,25}$ At a surfactant concentration of $0.9 \%$ in the oil surrounding the droplets and at $200 \mathrm{nM}$ kinesin-14 inside the droplet we observed, using confocal time-lapse fluorescence microscopy, the development of a dynamic vortex-like organisation of microtubules with the motor distributing along the microtubules, eventually becoming enriched towards the centre of the vortex (Fig. 2a and Movie 1, $\mathrm{ESI} \dagger$ ). This type of organisation is reminiscent of previous vortices observed when microtubules were organised by plus end directed motors, either in micro-fabricated chambers similar in diameter to our droplets ${ }^{10}$ or in the absence of confinement at low motor concentrations where motors are thought to reach microtubule ends less efficiently. ${ }^{11}$

To investigate whether vortex formation in our droplets has geometrical or biochemical origins, we doubled the kinesin-14 concentration to $400 \mathrm{nM}$ and observed now a self-organisation process more resembling aster formation, with a more concentrated motor accumulation in the centre of the aster (Fig. 2b). However, many microtubules appeared bent, suggestive of a potential geometrical effect on the aster structure. This might indicate that microtubules are very long, or that cross-linked microtubule bundles are too long to fit into the droplet. Individual microtubules are expected to be longer at lower nucleation rates because the free tubulin concentration decreases more slowly when fewer microtubules are growing, and parallel microtubule bundles are also expected to be longer at lower motor activities, because microtubule end-to-end sliding may be less efficient.

Next, we used a surfactant concentration of $1.8 \%$, double the value used above, in an attempt to increase the efficiency of microtubule nucleation. With a kinesin-14 motor concentration of $200 \mathrm{nM}$, we now observed relatively fast microtubule nucleation locally at the droplet boundary (Fig. 2c, $3.5 \mathrm{~min}$ ), possibly induced by a PEG density dependent protein adsorption effect. ${ }^{46}$ This was followed by the appearance of an apparently structureless microtubule/motor ring, indicative of a microtubule/motor shell in three dimensions. Remarkably, rather than remaining stuck to the boundary, this shell contracted, thereby dramatically reorganising the microtubules. Eventually an aster formed with microtubule minus ends focused into a single pole where the vast majority of
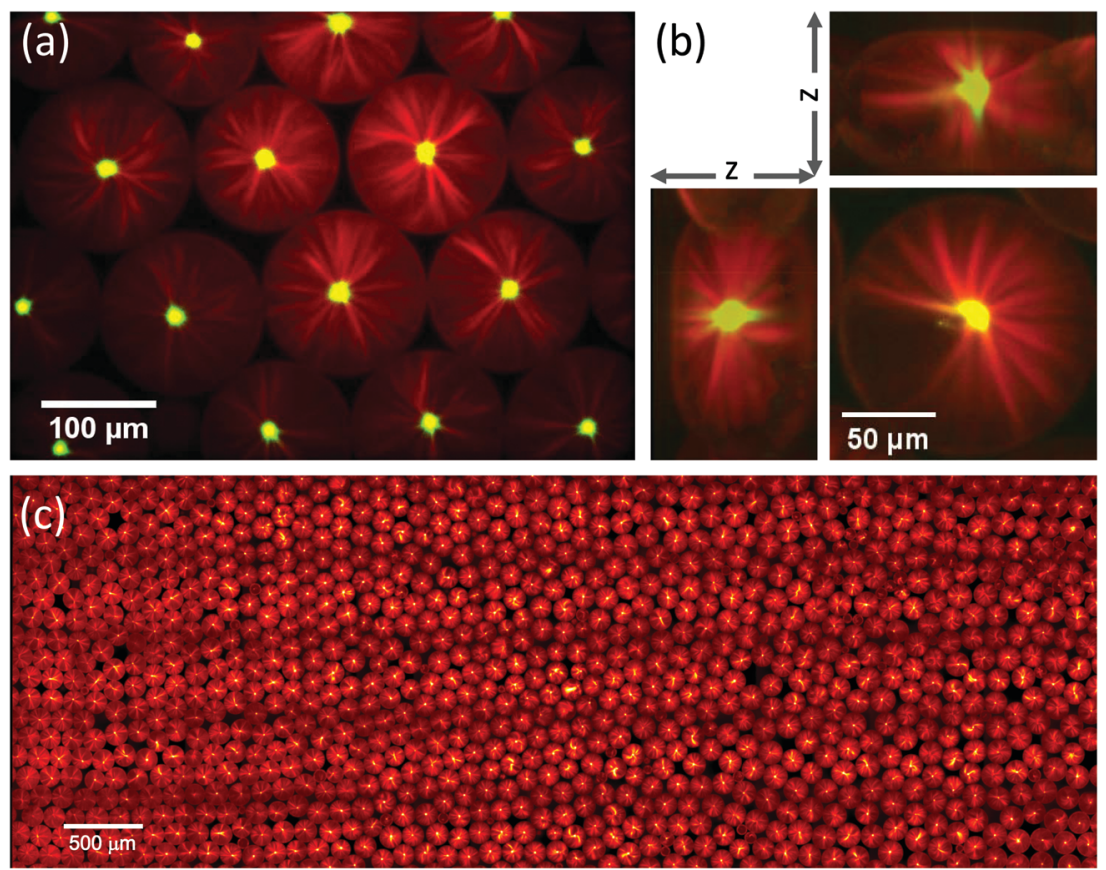

Fig. 3 Robust formation of centred microtubule asters in surfactant-stabilised droplets. Conditions as in Fig. 2c: $1.8 \%$ surfactant, 200 nM mCherrykinesin-14 (green), $40 \mu \mathrm{M}$ Alexa647-tubulin (red), $34^{\circ} \mathrm{C}$. (a) Confocal microscopy image of the central plane of individual isolated microtubule asters formed inside several droplets. (b) Slices through a z-stack generated from confocal images, showing the three-dimensional nature of the aster structure. (c) Montage of 95 images showing a region of the microscope slide $7.4 \times 2.7 \mathrm{~mm}$, and containing approximately 1400 droplets. Whole slide contains approximately 10000 droplets. 
(a)

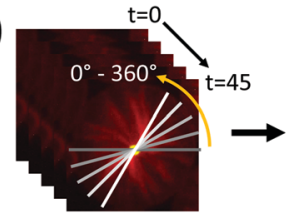

Single droplet time series

(b)

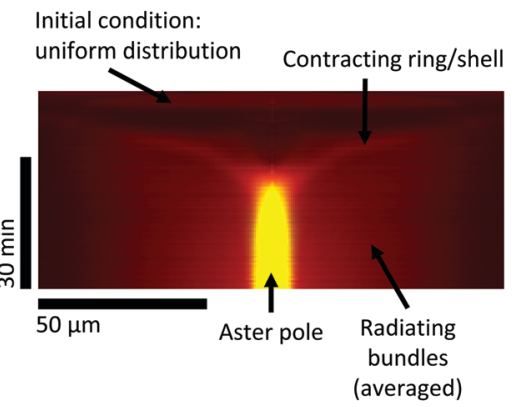

(d)

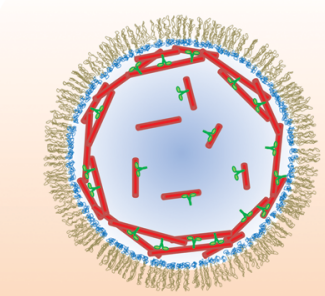

(i)

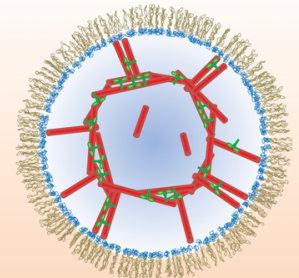

(ii) (c)
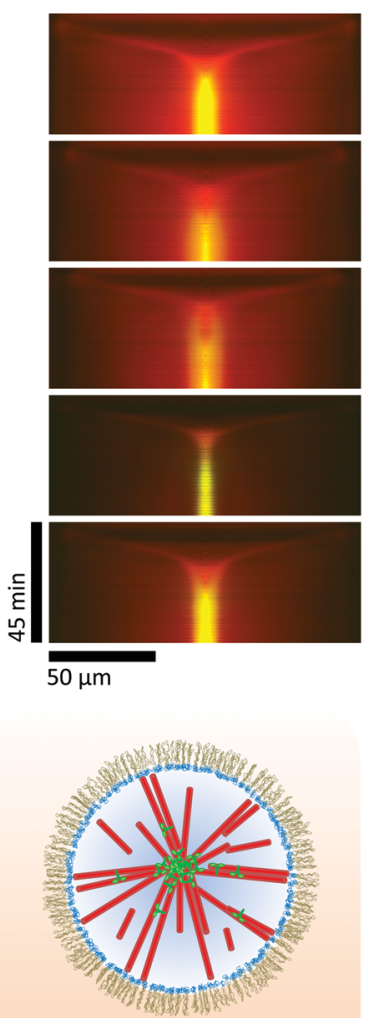

(iii)

Fig. 4 Kinetics of aster formation via contraction of a spherical network. (a) Illustration of the procedure for generating a radially averaged kymograph (space-time plot) from a time-lapse movie, to reveal how the average density of motors and microtubules measured along the droplet diameter develops over time. 360 kymographs are produced at $1^{\circ}$ intervals, and averaged together (Methods and Movie 4, ESI $\dagger$ ). (b) Typical averaged kymograph, showing the main features of the pathway of aster formation by a constriction mechanism. Kinesin-14 in green, tubulin in red. $t=0$ is at the top of the image. (c) Selection of five kymographs from different droplets at the same conditions (as in Fig. 2c and 3), showing that the essential features of the mechanism are retained. Colour code as in (b). (d) Schematic model: (i) microtubules nucleate preferentially at the boundary; (ii) molecular motors cross-link microtubules and slide them, causing contraction of the ring/shell; (iii) microtubule minus-ends sorted into a pole, stabilised by cross-linking motors.

motors strongly accumulated, while the microtubules radiated outward towards the droplet boundary as rather straight bundles (Fig. 2c). This mechanism of aster formation by contraction of a spherical network shell differs markedly from motor-driven microtubule aster formation pathways observed earlier ${ }^{9-11,25}$ and appears to be a direct consequence of confinement in combination with the more efficient microtubule nucleation at the droplet boundary under our conditions. This mechanism of aster formation also demonstrates that pole formation is a special case of three-dimensional network contraction.

Aster formation was remarkably robust. In the vast majority of observed cases, a single aster formed in each droplet, having its focal point well centred with respect to the droplet boundary, both in the $x / y$ plane (Fig. 3a) and the $z$ direction (Fig. 3b). Thousands of droplets, almost all of them with well centred selforganised microtubule asters could be reproducibly observed on a single slide (Fig. 3c), indicating a very strong preference for this configuration of our biochemical system when under confinement. This suggests that a well-controlled size of confinement can impose constraints on self-organising motor/microtubule systems to promote robustness of organisation, as is also required for the cytoskeleton in living cells.
To elucidate the characteristic features of the network constriction mechanism, we produced kymographs (spacetime plots) showing the temporal development of motor and microtubule densities measured along the droplet diameter (Fig. 4a, Movie 4 and Methods, ESI $\dagger$ ). Evidently, an initially uniform distribution leads within a few minutes to local microtubule nucleation at the boundary, followed by network constriction towards the droplet centre (Fig. 4b). Constriction slows down over time as the density of the spherical network increases, finally leading to a pole with strong motor accumulation (Fig. 4b), indicative of efficient polarity sorting of the now radiating microtubules. These characteristic features of the pathway are remarkably reproducible, as kymographs showing self-organisation in an arbitrary selection of five droplets show (Fig. 4c). These kymographs revealed that initially constriction caused the diameter of the network to shrink with a speed of $188 \pm 20 \mathrm{~nm} \mathrm{~s}^{-1}$, which is considerably faster than the typical kinesin-14 speed of $\sim 20 \mathrm{~nm} \mathrm{~s}^{-1}, 9$ demonstrating that multiple local contraction events are coupled leading to fast collective network constriction. Taken together, these observations demonstrate a novel and robust mechanism of motor-dependent microtubule aster formation (Fig. 4d). 


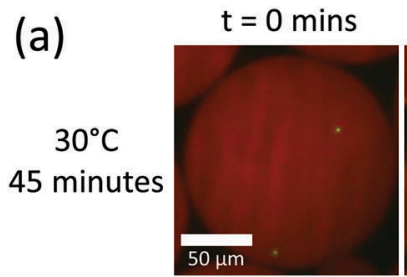

(b)

Finally, we investigated whether the constriction mechanism was a consequence of the process of droplet formation or an inherent property of the encapsulated biochemical system. To address this question, we asked whether aster formation via the shell contraction mechanism can be repeated after aster disassembly. We first allowed asters to form in droplets at $30{ }^{\circ} \mathrm{C}$, showing again aster formation by network constriction (Fig. $5 \mathrm{a}$ and Movie 5 - part 1, ESI $\uparrow$ ). We then induced microtubules to depolymerise by cold treatment (Methods), causing the asters to disappear, leaving only a small remnant of the aster centre where the protein concentration was highest, while most tubulin and motors redistributed throughout the droplet (Fig. 5b and Movie 5 - part 2, ESI $\dagger$ ). Reheating the flow chamber initiated microtubule polymerisation again, and a second round of aster formation began, again by the constriction mechanism (Fig. $5 \mathrm{c}$ and Movie 5 - part 3, ESI $\dagger$ ). This conclusion was confirmed by a 'funnel'-shaped congression of high motor and microtubule densities in the kymographs, indicative of spherical network constriction and pole formation (Fig. 5d, left and right, respectively). These observations demonstrate the dynamic steady state nature of the self-organised aster, in contrast to a static structure formed by an irreversible process. This demonstrates also that the biochemical conditions here allow the preservation of enzyme activities for well over an hour in our droplet system. Moreover, the repeatability of aster formation demonstrates that the constriction mechanism is an intrinsic property of the system characterised by its (bio)chemical composition and its geometrical properties, in contrast to a historydependent process retaining some memory from droplet formation.

\section{Conclusions}

We have developed a new and robust experimental system for motor/microtubule self-organisation in surfactant-stabilised droplets of well-defined size. The experimental system preserved protein activities well, allowing efficient nucleation of dynamic microtubules in the absence of artificial microtubule stabilisers. Choosing a large droplet diameter of about $145 \mu \mathrm{m}$ allowed us to study the conditions of reproducible microtubule aster formation in thousands of almost identical droplets. The pathway of self-organisation depended on both the motor concentration, as expected, ${ }^{9-11}$ and on the chemical properties 
of the boundary. Under optimal conditions, single asters formed robustly via spherical network constriction, a mechanism that was notably different from previous aster formations in unconfined geometries. $^{9-11}$ The constriction pathway illustrates the close relationship between active network contraction and aster formation. It is particularly interesting to note the continuous topological transition whereby a spherical network of randomly oriented filaments is reorganised by constriction to form a point from which polarity-sorted bundles radiate.

Compared to previous biochemical self-organisation experiments with purified motors and microtubules without confinement ${ }^{9-11}$ or within droplets of uncontrolled size, ${ }^{25}$ the pathway of aster formation was highly reproducible and the size of the asters was uniform. The radial symmetry of asters filling the entire droplet volume led to reliable concentration of the vast majority of microtubule minus ends and minus-end-directed motors in the droplet centre, demonstrating the ability of the system to find its centre with high fidelity. The additional constraint of imposing confinement of well-controlled size confers robustness to the outcome of self-organisation, which may well be of relevance for the self-organising cytoskeleton in living cells.

It is also interesting to see that microtubules and cross-linking kinesins can form a constricting network that resembles to a certain extent the contraction of the cytokinetic ring consisting of actin filaments and myosin motors that assembles at the cell boundary to separate the two daughter cells during cell division. ${ }^{4748}$ The outcome of constriction is different in these cases due to differences in the properties of the filaments and motors, with the cytokinetic ring collapsing without a topological change to form a compact midbody, whereas the microtubules in our experiments undergo a topological transition to form a large aster of bundles tethered to a well-defined central pole. Despite the different final structures described, the use of symmetrical boundary conditions to determine the eventual organisation of a dynamic system can be seen as a fundamental design principle for micro-scale active matter selfassembly.

\section{Author contributions}

M. J. performed all experiments and data analysis. M. W. made the droplet-based microfluidic devices. M. J. and T. S. designed the study. M. J. and T. S. wrote the manuscript with input from M. W., I. P. and J. S. assisted with the polymer stabilized droplet microfluidics. All authors contributed to discussions and to editing the manuscript.

\section{Conflicts of interest}

There are no conflicts to declare.

\section{Acknowledgements}

We thank Hella Baumann and Claire Thomas for help with protein expression and purification, Nicholas I. Cade for microscopy support and help with data analysis, and Jonathon Hannabuss for useful discussions. This work was supported by the Francis Crick Institute which receives its core funding from Cancer Research UK (FC001163), the UK Medical Research Council (FC001163), and the Wellcome Trust (FC001163). T. S. acknowledges funding also from the European Research Council (Advanced Grant, project 323042). The work was also supported by the MaxSynBio initiative of the Max Planck Society. J. P. S. is the Weston Visiting Professor at the Weizmann Institute of Science and part of the excellence cluster CellNetworks at the University of Heidelberg. Parts of the research leading to these results have received funding from the European Research Council/ERC Grant Agreement no. 294852, SynAd. In general, support of the Max Planck Society is highly appreciated.

\section{References}

1 K. J. Helmke, R. Heald and J. D. Wilbur, Int. Rev. Cell Mol. Biol., 2013, 306, 83-125.

2 T. M. Kapoor, Biology, 2017, 6, 8.

3 D. Needleman and Z. Dogic, Nat. Rev. Mater., 2017, 2, 1-14.

4 E. J. Peterman and J. M. Scholey, Curr. Biol., 2009, 19, R1089-R1094.

5 E. Karsenti, F. Nedelec and T. Surrey, Nat. Cell Biol., 2006, 8, 1204-1211.

6 T. J. Keating and G. G. Borisy, Biol. Cell., 1999, 91, 321-329.

7 A. D. Sanchez and J. L. Feldman, Curr. Opin. Cell Biol., 2017, 44, 93-101.

8 M. Dogterom and T. Surrey, Curr. Opin. Cell Biol., 2013, 25, 23-29.

9 C. Hentrich and T. Surrey, J. Cell Biol., 2010, 189, 465-480.

10 F. J. Nedelec, T. Surrey, A. C. Maggs and S. Leibler, Nature, 1997, 389, 305-308.

11 T. Surrey, F. Nedelec, S. Leibler and E. Karsenti, Science, 2001, 292, 1167-1171.

12 T. Torisawa, D. Taniguchi, S. Ishihara and K. Oiwa, Biophys. J., 2016, 111, 373-385.

13 G. Henkin, S. J. DeCamp, D. T. Chen, T. Sanchez and Z. Dogic, Philos. Trans. R. Soc., A, 2014, 372, 20140142.

14 T. Sanchez, D. T. Chen, S. J. DeCamp, M. Heymann and Z. Dogic, Nature, 2012, 491, 431-434.

15 T. Sanchez, D. Welch, D. Nicastro and Z. Dogic, Science, 2011, 333, 456-459.

16 P. J. Foster, S. Furthauer, M. J. Shelley and D. J. Needleman, eLife, 2015, 4, e10837.

17 K. Suzuki, M. Miyazaki, J. Takagi, T. Itabashi and S. Ishiwata, Proc. Natl. Acad. Sci. U. S. A., 2017, 114, 2922-2927.

18 M. Elbaum, D. Kuchnir Fygenson and A. Libchaber, Phys. Rev. Lett., 1996, 76, 4078-4081.

19 D. K. Fygenson, J. F. Marko and A. Libchaber, Phys. Rev. Lett., 1997, 79, 4497-4500.

20 M. Cosentino Lagomarsino, C. Tanase, J. W. Vos, A. M. Emons, B. M. Mulder and M. Dogterom, Biophys. J., 2007, 92, 1046-1057.

21 C. Faivre-Moskalenko and M. Dogterom, Proc. Natl. Acad. Sci. U. S. A., 2002, 99, 16788-16793.

22 L. Laan, N. Pavin, J. Husson, G. Romet-Lemonne, M. van Duijn, M. P. Lopez, R. D. Vale, F. Julicher, S. L. Reck-Peterson and M. Dogterom, Cell, 2012, 148, 502-514. 
23 M. Vleugel, S. Roth, C. F. Groenendijk and M. Dogterom, J. Visualized Exp., 2016, DOI: 10.3791/54278.

24 F. C. Keber, E. Loiseau, T. Sanchez, S. J. DeCamp, L. Giomi, M. J. Bowick, M. C. Marchetti, Z. Dogic and A. R. Bausch, Science, 2014, 345, 1135-1139.

25 H. Baumann and T. Surrey, J. Biol. Chem., 2014, 289, 22524-22535.

26 M. Pinot, F. Chesnel, J. Z. Kubiak, I. Arnal, F. J. Nedelec and Z. Gueroui, Curr. Biol., 2009, 6, 6.

27 I. Platzman, J. W. Janiesch and J. P. Spatz, J. Am. Chem. Soc., 2013, 135, 3339-3342.

28 M. Castoldi and A. V. Popov, Protein Expression Purif., 2003, 32, 83-88.

29 A. Hyman, D. Drechsel, D. Kellogg, S. Salser, K. Sawin, P. Steffen, L. Wordeman and T. Mitchison, Methods Enzymol., 1991, 196, 478-485.

30 C. E. Walczak, S. Verma and T. J. Mitchison, J. Cell Biol., 1997, 136, 859-870.

31 P. B. Umbanhowar, V. Prasad and D. A. Weitz, Langmuir, 2000, 16, 347-351.

32 B. Bohl, R. Steger, R. Zengerle and P. Koltay, J. Micromech. Microeng., 2005, 15, 1125-1130.

33 D. Qin, Y. N. Xia and G. M. Whitesides, Adv. Mater., 1996, 8, 917-919.

34 D. C. Duffy, J. C. McDonald, O. J. A. Schueller and G. M. Whitesides, Anal. Chem., 1998, 70, 4974-4984.
35 G. M. Whitesides, E. Ostuni, S. Takayama, X. Y. Jiang and D. E. Ingber, Annu. Rev. Biomed. Eng., 2001, 3, 335-373.

36 S. Takeuchi, P. Garstecki, D. B. Weibel and G. M. Whitesides, Adv. Mater., 2005, 17, 1067-1072.

37 P. A. Zhu and L. Q. Wang, Lab Chip, 2017, 17, 34-75.

38 P. Bieling, I. A. Telley, C. Hentrich, J. Piehler and T. Surrey, Methods Cell Biol., 2010, 95, 555-580.

39 R. Roy, S. Hohng and T. Ha, Nat. Methods, 2008, 5, 507-516.

40 S. Tyagi, V. VanDelinder, N. Banterle, G. Fuertes, S. Milles, M. Agez and E. A. Lemke, Nat. Methods, 2014, 11, 297-300.

41 J. W. Janiesch, M. Weiss, G. Kannenberg, J. Hannabuss, T. Surrey, I. Platzman and J. P. Spatz, Anal. Chem., 2015, 87, 2063-2067.

42 H. Baumann and T. Surrey, Methods Cell Biol., 2015, 128, 39-55.

43 H. B. McDonald, R. J. Stewart and L. S. Goldstein, Cell, 1990, 63, 1159-1165.

44 R. A. Walker, E. D. Salmon and S. A. Endow, Nature, 1990, 347, 780-782.

45 G. Fink, L. Hajdo, K. J. Skowronek, C. Reuther, A. A. Kasprzak and S. Diez, Nat. Cell Biol., 2009, 11, 717-723.

46 P. Katira, A. Agarwal and H. Hess, Adv. Mater., 2009, 21, 1599-1604.

47 B. Mierzwa and D. W. Gerlich, Dev. Cell, 2014, 31, 525-538. 48 T. D. Pollard, Biophys. J., 2014, 107, 2499-2507. 